\title{
A Relativistic Escape Velocity Maximum of Light Speed
}

\author{
David Grant Taylor \\ Edmonton, Canada \\ Email:dgtaylor@telusplanet.net
}

Received 17 May 2016; accepted 4 July 2016; published 8 July 2016

Copyright (C) 2016 by author and Scientific Research Publishing Inc.

This work is licensed under the Creative Commons Attribution International License (CC BY). http://creativecommons.org/licenses/by/4.0/

\section{(c) (i) Open Access}

\section{Abstract}

There are parallels between the time distortion equations of General and Special Relativity. The time distortion in Special Relativity limits the "Real" velocity of a particle to the speed of light $|c|$ by slowing the velocity of bosons/energy and increasing the mass of fermion||lepton (or matter) particles. In General Relativity, the gravitational slowdown of bosons/gravitons limits the escape velocity $\left|v_{E s c}\right|$ to light speed. $\left|v_{E s c}=(2 G M / r)^{0.5}\right|$ can also be written as $\left|v_{E s c}^{2}=2 G M / r\right|$. So an alternate grammar for the equation could be

$$
\begin{gathered}
\text { Time }^{\prime}=\text { Time } /\left(1-2 G M / r c^{2}\right)^{0.5} \\
\text { Time }^{\prime}=\text { Time } /\left(1-(2 G M / r) * 1 / c^{2}\right)^{0.5} \\
\text { Time }^{\prime}=\text { Time } /\left(1-\left(v_{E s c}^{2}\right) * 1 / c^{2}\right)^{0.5} \\
\text { Time }^{\prime}=\text { Time } /\left(1-v_{E s c}^{2} / c^{2}\right)^{0.5}
\end{gathered}
$$

The above contests currently held properties of Schwarzschild Objects. Relativistic theory predicts the slowdown of gravitons/bosons, while their velocity only approaches zero. The different mathematical logic of Gravitational Force $\|G F\| G F=G M m / r^{2}$ would mean no limit on the force. Matter formed through compression of bosons can escape after formation by absorbing kinetic energy from the slowing photons/bosons in its surroundings. So matter formation can occur via a steady-state mechanism. The limit on $v_{E s c}$ allows matter to escape in events that parallel both the Big Bang and Cyclic cosmology. Additional arguments are made as to the validity of relativity.

\section{Keywords}

Relativity, Escape, Velocity, Gravitation, Force, Nuclear Decay, Schwarzschild Limit, Antimatter, 


\section{Sagittarius A}

\section{Light Speed Limits for Escape Velocities}

In a Schwarzschild Object (SO), boson slowdown and gravitational pressure can transform energy into fermion\|. lepton (or matter) particles. Parallels between the time distortion equations in General Relativity (GR) and Special Relativity (SR) can be reasoned. Just as SR limits "Real” velocity to $c$, parallels can the reasoned with the combination of the escape velocity and GR Time equations to establish aescape velocity $\left|v_{E s c}\right|$ limit of $c$. Energetic matter particles can escape when bosons slow to below the speed of light. Escape velocity is determined by

$$
v_{E s c}=(2 G M / r)^{0.5}
$$

That can also be written as

$$
v_{E s c}^{2}=2 G M / r
$$

So GR time distortion can be rewritten

$$
\begin{gathered}
\text { Time }^{\prime}=\text { Time } /\left(1-2 G M / r c^{2}\right)^{0.5} \\
\text { Time }^{\prime}=\text { Time } /\left(1-(2 G M / r) * 1 / c^{2}\right)^{0.5} \\
\text { Time }^{\prime}=\text { Time } /\left(1-\left(v_{E s c}^{2}\right) * 1 / c^{2}\right)^{0.5} \\
\text { Time }^{\prime}=\text { Time } /\left(1-v_{E s c}^{2} / c^{2}\right)^{0.5}
\end{gathered}
$$

Observations of muon decay [1] confirm Relativistic time slowdown. All bosons are slowed.

GR gravitational distortion must slow gravitons, limiting $v_{E s c}$ to the speed of light. Equation (2) is not a new equation derived from GR time distortion but a rephrasing of the original. Using SR logic, $\left|1-2 G M / r c^{2}\right|$ will never reach a value of zero. Passage of time will never cease, only approach cessation.

Because of the different grammar for Gravitational Forces [GF]

$$
G F=G M m / r^{2}
$$

Gravitational force will always have a Real value. The number of different vectors to those forces is only limited by the number of bodies of matter/energy in the Local observable Universe. If our Local Universe were part of an Infinite Cosmos, there would be no limit on those vectors or their force. The spontaneous coagulation of energy/matter to the density that is required for a white hole to form can therefore occur. The likelihood of such an occurrence depends on the concentration of energy in the location of interest. A sufficient density of energy/matter can spontaneously coagulate to the density needed for a white hole to form. No special event is necessary, only the passage of sufficient time.

\section{Consequences of White Hole Formation}

The spontaneous formation of a White hole in a pure energy environment is improbable. Concentration of energy in a defined sphere to a degree comparable to that in a neutron star or pulsar may have an infinitesimal probability, but not zero. Remove all time constraints and it is theoretically possible for a white hole to form spontaneously. The improbability of such an event is unknowable. Density of the Cosmic Microwave Background Radiation (CMBR) has only been confirmed to be homogeneous in space-time observations since the release of "A Measurement of Excess Antenna Temperature at $4080 \mathrm{Mc} / \mathrm{s}$ " [2]. The progression of time before the Big Bang or the creation of space is beyond what can be observed: "No one knows how the first space, time, and matter arose" [3]. The presumption that all three arose from absolute nothingness is an unreasonable postulate. However, the existence of an unknown expanse of a cosmos with an undeterminable direction of event progression, which contained an unknowable matter/energy substance, is a worthwhile conjecture. Reality had to come from somewhere. 
Current theory begins with the hatching of a "Cosmic Egg” (CE) into a Big Bang. One suggestion for how such a CE could have formed is the expansion reversal of a previous Universe, although this offers no explanation of for the first CE that formed an infinite energy-filled reality. So let us presume the spontaneous formation and compression of an SO or white hole, though unlikely, is more probable for an object with dimensions below those of current CE theory. The action required would be undeniably fundamental, possibly Googol scale improbability, nonetheless inevitable in an infinite reality. It would be the spontaneous congregation of sufficient Photon or Boson particles to form an SO. Following the formation of this object, gravitational and relativistic effects would lead to the continual acquisition of more energy bosons [4] and their continual slowdown, resulting in a matter-free area where a spontaneous boson concentration formed an absolute white hole capturing a theoretically limitless number bosons with gravitational forces of the SO. This object would expand; capturing more Relativistically or gravitationally slowed bosons. Gravitational effects would force central compression of the expanding ball of energy. The equations presented above argue for a $c$ limit to escape velocity but significant boson retention.

Because of rising mass, gravity would increase at more central points in the White Hole, compressing captured bosons more and more. Gravitational force would be distorted downwards, though not with the limits as those on escape velocity. Relativistic slowdown and gravitational force would compress and slow the bosons until they newly formed matter, with matter and antimatter formation initially being equally probable. It is a reasonable postulate that once a significant proportion of either matter or antimatter had formed there could be a nuclear catalytic effect that would drive the production of more particles of the same type. Matter particles that had been captured or manufactured by the white hole would acquire energy from all free Bosons; leading eventually to sufficient kinetic energy to reach the escape velocity. The alternative possibility is the current theory that spontaneous and random formation in an expanding body of pure energy led to the current predominance of matter. If they were formed while separating from one another it is unreasonable to postulate that they could have any interaction to promote a particular flavour. In the calamitous experiments at the CERN, it is estimated that upon creation any antimatter created in a free state lasted 4.0E-10 seconds [5]. There were later experiments that kept anti-Hydrogen atoms with a lifetime of as much as 1000 seconds, but that was in a very artificial "trapping" of the atoms and not allowing contact with matter particles. The current model of the Big Bang, with a expansion of all reality at hyper-relativistic velocities can be argued to be the most pro-entropic moments in all of visible reality —or the absolute antithesis of the above experiment.

Non-black SOs such as the bright Abell 2261 galaxy cluster support this argument. If the brightness of such objects is primarily caused by the descent of captured matter, then the signal should be red-shifted by gravity because of the withdrawal velocity of the captured matter away from observers and the strong relativistic effects at the object border. An excellent image of the extremely bright Abell 2261 object is available at the NASA website [6]: http://www.spacetelescope.org/images/heic1216a/.

Bosons are a potential candidate for the fundamental state of reality because valid mechanisms for their conversion into matter particles through relativistic forces are available. Both SR and GR offer potential mechanisms for the generation of matter. The CMBR serves as simple and compelling evidence of the universality of bosons as the source of matter. The proposition that the normal state of our cosmos is as a dispersed collection of pure energy is more consistent with the principle of entropy than is the order that the existence of a single matter particle would introduce. The most orderly, least entropic object in our understanding of the history of our local, visible Universe is arguably the singularity (regardless of its dimensions) that exploded in the first Big Bang.

How the formation of that singularity came about is still a topic of much debate, so no attempt will be made to credit (or discredit) any of the extant propositions. So a question posed to the reader: which is the more reasonable hypothesis on formation of the finite space around us into our local universe?

1) An infinite expanse with a relativistic mechanism for the conversion of energy into matter. In an extremely unlikely circumstance, a block of that energy becomes sufficiently concentrated to form an SO. The probability of this occurrence is unimportant because the expanse in question is infinite in time, space and energy. Either flavour of matter or antimatter acts as a catalyst for the formation of more of the same. The only available evidence of the finite nature of our local universe consists of observations made over an extremely tiny portion of the time span proposed by current theory. They were drawn from an infinitesimal portion of the space that exists in our reality. The newly formed matter or antimatter could eventually acquire sufficient kinetic energy to completely escape because the escape velocity would always be below the speed of light. 
-Or-

2) Explosion at a hyper-relativistic speed of a currently unobserved object within a finite expanse, with no confirmed mechanisms for its formation or causes for its explosion. Observations of those finite limits have broadened throughout the entire history of human thinking and science.

The SR time distortion expression, | Time' $=$ Time $/\left(1-v^{2} / c^{2}\right)^{0.5} \mid$, is currently understood to dictate the slowing of time. Relativistic velocity shift imposed on photons has been experimentally confirmed [7]. Relativistic increases in mass of a matter object approaches infinity as the velocity of that object approaches $c$. So the fundamental properties of matter (both nuclear and chemical) would change as the atomic mass of the individual atoms changes in proportion to their atomic number. To the same degree, the forces/energy that dictate the reactions among nucleons, atoms, and/or molecules should slow and weaken.

The principle hydrogen isotope, ${ }^{1} \mathrm{H}$, has an atomic mass of 1.007825 [8]. An element with profoundly different nuclear structure, the principle iron isotope, ${ }^{26} \mathrm{Fe}$, has an atomic mass of 55.934939 [9]. A 2.59627884E8 m/s velocity brings an approximate distortion factor of 2.0. At that velocity the mass of each individual nucleon would double, with the mass of each boson being halved. Would the interactions of ${ }^{1} \mathrm{H}$, with an atomic mass of 2.015650 ( or ${ }^{26} \mathrm{Fe}$, with an atomic mass of 111.8699), remain the same but slower? Deuterium has a mass equal to approximately double that of ${ }^{1} \mathrm{H}$, but a distorted ${ }^{1} \mathrm{H}$ would not be exactly identical to an undistorted ${ }^{2} \mathrm{H}$. Distorted hydrogen atoms would also have slowed electrons. No isotope of iron exists with double the mass of the principle isotope. The combination of the increased mass of particles and the slowing/weakening of the bosons that maintain their structure (the repulsive force of positive charges in the nucleus and the binding strong nuclear force of gluons) could lead to the breakdown of heavier nuclides into elements of lower atomic numbers. How absolute or simplistic this breakdown would be is not currently known, but what be said with some certainty is that matter particles would increase in mass through absorption of the lessening energy of the forces maintaining its structure would be less stable.

So an argument against current views of GR effects: the increase in mass of matter particles would not be of the same proportionality as SR distortion, though an increase would occur because of boson slowdown. Structure of nucleons, atoms, and molecules originate from interactions between their matter masses and forces carried by gravitons, photons, gluons, and $\mathrm{X}$ and $\mathrm{Y}$ bosons around them. Increases in matter particles mass and slowdown of all bosons would result in a fundamental change. Conservation of energy argues a parallel increase in the number of gravitons. But they would move with half the velocity of undistorted gravitons. Energy is a function of force over a given time. Because of the slowed pace of bosons, fewer of them would interact over the same time period than the undistorted flavour. For any given time period, there would be less energy. So the escape velocity and the force of gravity would degrade from what they were with no distortion.

\section{Estimating Escape Velocity Limits Based on an Observed Phenomenon}

A limit on the escape velocity is estimated here based on the current Schwarzschild equations and the parameters of a known SO to illustrate the incompleteness and inconsistency of those equations. The mass values of the relevant objects are assumed precise to 50 decimal places. While imprecise in almost all cases, it is necessary for more valid illustration of certain theoretical principles, including those in this paper. These principles do not require precision for their establishment, simply consistency. So presumed are themass of the Sun [10] $\left[\right.$ Solar $\left._{\text {Mass }}=1.9885 \sim 00 \mathrm{E} 30 \mathrm{~kg}\right]$, the Gravitational Constant [9] $\left[G=6.67384800 \sim 00 \mathrm{E} \mathrm{m}^{3} \cdot \mathrm{kg}^{-1} \cdot \mathrm{s}^{-2}\right]$ and the Planck Length [11] [lp =1.61629900 00E - $35 \mathrm{~m}]$.

The SO with the largest mass in our galaxy is assumed to be Sagittarius A [12] (SA), which formed at the core of the Milky Way, with a right ascension of 17 h $45 \mathrm{~m} 40.0409$ s, a declination of -29²'28.118" with a mass [13] $\left[\mathrm{SA}_{\text {Mass }}\right]$ is 4.31 million times that of our Sun. The SA object used here is an illustration, not as a theoretical proof.

$$
\begin{gathered}
\mathrm{SA}_{\text {Mass }}=4.3100 \sim 00 \mathrm{E} 6 * \text { Solar }_{\text {Mass }} \\
\mathrm{SA}_{\text {Mass }}=8.5706505 \sim 00 \mathrm{E} 36 \mathrm{~kg}
\end{gathered}
$$

The SA object Schwarzschild radius $\left[\mathrm{SA}_{\text {Schwarz }}\right]$ determined from those presumptions:

$$
\mathrm{SA}_{\text {Schwarz }}=2 * G * \mathrm{SA}_{\text {Mass }} / c^{2}
$$




$$
\begin{gathered}
\mathrm{SA}_{\text {Schwarz }}=\left(2 * 6.67384800 \sim 00 \mathrm{E}-11 \mathrm{~m}^{3} \cdot \mathrm{kg}^{-1} \cdot \mathrm{s}^{-2} * 8.5706505 \sim 00 \mathrm{E} 36 \mathrm{~kg}\right) /(299792458 \mathrm{~m} / \mathrm{s})^{2} \\
\mathrm{SA}_{\text {Schwarz }}=1.27285275203225548390675120034832177678110953867744 \mathrm{E} 10 \mathrm{~m}
\end{gathered}
$$

In classical relativistic theory, the Time distortion||Time_Dist at the exact border of the SO is infinite. We assume an ideal of a single non-relativistic second. Time distortion at 1 Planck length||lp (1.61629900 00E-35 m [14]) beyond the SO border of the SA object $\left[\mathrm{SA}_{-} \mathrm{Time}_{\mathrm{SO}+l \mathrm{p}}\right]$ :

$$
\mathrm{SA}_{-} \text {Time }_{\mathrm{SO}+\mathrm{lp}}=1 /\left(1-2 * G * \mathrm{SA}_{\text {Mass }} /\left(\mathrm{SA}_{\mathrm{Schwarz}}+\mathrm{lp}\right) * c^{2}\right)^{0.5}
$$

$$
\begin{aligned}
\text { SA_Time } & =1 /\left(1-2 * 6.67384800 \sim 00 \mathrm{E}-11 \mathrm{~m}^{3} \cdot \mathrm{kg}^{-1} \cdot \mathrm{s}^{-2} * \sim\right. \\
& \left.8.15305500 \sim 00 \mathrm{E} 36 \mathrm{~kg} /(1.272 \sim 7744 \mathrm{E} 10 \mathrm{~m}+1.61629900 \sim 00 \mathrm{E}-35) * \mathrm{c}^{2}\right)^{0.5}
\end{aligned}
$$

$$
\text { SA_Time }{ }_{\mathrm{SO}+\mathrm{lp}}=2.80632286920760473419538643218572986251919661087977 \mathrm{E} 22 \mathrm{~m}
$$

The time distortion at plus 1.0 metre $\left[\mathrm{SA}_{-} \mathrm{Time}_{\mathrm{SO}+1}\right]$ :

$$
\begin{aligned}
\text { SA_ } & \text { Time }_{\text {SO }+1}=1 /\left(1-2 * G * \mathrm{SA}_{\text {Mass }} /\left(\mathrm{SA}_{\text {Schwarz }}+1.00 \sim 00 \mathrm{~m}\right) * c^{2}\right)^{0.5} \\
\mathrm{SA}_{-} \text {Time }_{\mathrm{SO}+1}= & \left(1-2 * 6.67384800 \sim 00 \mathrm{E}-11 \mathrm{~m}^{3} \cdot \mathrm{kg}^{-1} \cdot \mathrm{s}^{-2} *\right. \\
& \left.8.15305500 \sim 00 \mathrm{E} 36 \mathrm{~kg} /(1.272 \sim 7744 \mathrm{E} 10 \mathrm{~m}+1.0 \mathrm{E} 00 \sim 00) * c^{2}\right)^{0.5} \\
\text { SA_Time }_{\mathrm{SO}+1}= & 1.12820776106719611765825987883803392470998556057620 \mathrm{E} 5
\end{aligned}
$$

The Ratio between these distortions: [RATIO $\left.{ }_{\text {Dist }}\right]$

$$
\begin{gathered}
\text { RATIO }_{\text {Dist }}=\text { SA }_{-} \text {Time }_{\text {SO+lp }} / \text { SA }_{-} \text {Time }_{\text {SO+1 }} \\
\text { RATIO }_{\text {Dist }}=7.49067 \sim 00961 \mathrm{E} 44 / 1.128 \sim 7620 \mathrm{E} 5 \\
\text { RATIO }_{\text {Dist }}=2.20475060018255306932356205067543965143504537301659 \mathrm{E} 12
\end{gathered}
$$

Comparing the $\mathrm{SA}$ gravitational forces $\left[\mathrm{SA}_{-} \mathrm{GF}_{\text {Schwarz }}\right] 1$ Planck length beyondthe $\mathrm{SA}_{\text {Schwarz }}$ border as above

$$
\mathrm{SA} \_\mathrm{GF}_{\text {Schwarz }}=G * \mathrm{SA}_{\text {Mass }} / \mathrm{SA}_{\text {Schwarz }}^{2}
$$

SA_GF Schwarz $=\left(6.6738480 \sim 0 \mathrm{E}-11 \mathrm{~m}^{3} \cdot \mathrm{kg}^{-1} \cdot \mathrm{s}^{-2}\right) /(1.21068 \sim 26421 \mathrm{E} 10 \mathrm{~m}+1.6161999700 \sim 00 \mathrm{E}-35 \mathrm{~m})^{2}$

$$
\text { SA_ } \text { FF }_{\text {Schwarz }}=3.7117722748901995864567774171441701737277653584 \mathrm{E} 6 \mathrm{~m} / \mathrm{s}^{2}
$$

One metre farther from the border the force $\left[\mathrm{SA}_{-} \mathrm{GF}_{\text {Schwarz }+1 \mathrm{~m}}\right]$ would be:

$$
\begin{aligned}
& \mathrm{SA}_{-} \mathrm{GF}_{\text {Schwarz }+1 \mathrm{~m}}=G * \mathrm{SA}_{\text {Mass }} /\left(\mathrm{SA}_{\text {Schwarz }}+1.00 \sim 00 \mathrm{E} 0\right)^{2} \\
& \text { SA _ GF } \text { Schwarz }+1 \mathrm{~m}=6.6738480 \sim 0 \mathrm{E}-11 \mathrm{~m}^{3} \cdot \mathrm{kg}^{-1} \cdot \mathrm{s}^{-2} * 8.1530550 \sim 0 \mathrm{E} 36 \mathrm{~kg} / \sim \text { ? } \\
& (1.21068 \sim 26421 \mathrm{E} 10 \mathrm{~m}+1.000 \sim 000 \mathrm{~m})^{2} \\
& \text { SA_GF } F_{\text {Schwarz+1 m }}=3.7117722742770291149029886053851463456506480279 \mathrm{E} 6 \mathrm{~m} / \mathrm{s}^{2}
\end{aligned}
$$

The difference $\left[G F_{D i f f}\right]$ between these two force values:

$$
\begin{gathered}
\mathrm{GF}_{\text {Diff }}=\mathrm{SA}_{-} \mathrm{GF}_{\text {Schwarz }}-\mathrm{SA}_{-} \mathrm{GF}_{\text {Schwarz }+1 \mathrm{~m}} \\
\mathrm{GF}_{\text {Diff }}=3.71177 \sim 43664 \mathrm{E} 6 \mathrm{~m} / \mathrm{s}^{2}-3.71177 \sim 91866 \mathrm{E} 6 \mathrm{~m} / \mathrm{s}^{2} \\
\mathrm{GF}_{\text {Diff }}=6.13170471553788811759023828077117340428362310702818 \mathrm{E}-4 \mathrm{~m} / \mathrm{s}^{2}
\end{gathered}
$$

So current theory contends that a difference in gravitational force of $6.131 \sim 818 \mathrm{E}-4 \mathrm{~m} / \mathrm{s}$ is reasonable. That is inconsistent with the distortion variance between those border points: an infinite distortion at the border, 2.806 
977E22 at 1 Planck Length and $1.1282 \sim 620 \mathrm{E} 5$ at one 1 metre. A Planck length within the border the distortion becomes imaginary, though what that means in Quantum level gravity issues has not been determined.

These numbers add to the mathematic reasoning of a maximum escape velocity for any body is $|c|$-speed of light-whatever its mass or radius. The GR escape velocity limit parallels the SR velocity limit and has notable implications for the mechanisms and dimensions for a Big Bang, a Cyclic Cosmology or a Steady-State reality. This writer contests almost all of the current estimates regarding the dimensions of the first Big Bang, not because it would be impossible for events at such a scale to occur but rather because their occurrence is simply extremely unlikely. These issues are argued much more cogently and specifically in other papers. The principal point of this paper is to argue for the existence of different equations in addition to current relativistic theory, which are exactly mathematically consistent with the originals.

The consequences of the above argument regarding the SA object would be that the combination of its gravitational pull and its relativistic distortion of photons and bosons would imply that it would capture more energy than it would expel. The captured photons/bosons would be drawn increasingly close to the centre. The increased relativistic effects would cause the photons/bosons to slow down, and conservation of energy would cause them to multiply in number.

\section{Matter/Antimatter Production through Compression and Nuclear Catalytic Effects}

It is known that the creation of antimatter particles can occur through the collision of matter particles. So a combination of energy (the kinetic energy of the colliding particles) and subatomic particles can produce both antimatter and matter particles. The matching production of matter and anti matter particles is not exactly determinate because of the mass and size of the antimatter particles produced. Though it is absolutely determinate that they are produced in comparable amounts because of the energy produced, experimental verification of the number of actual matter particles in a beam can never be achieved because of uncertainty effects.

The key point to note from the above is that the only currently known methods for creating anti-particles are relatively violent. The production of matter (or antimatter) through compression has never been observed. However, a method of doing so can be theorized. In particle collisions, immediately after collision, any produced particles separate at relativistic velocities. They have no interaction other than in that collision.

Consider the alternative: pure energy is compressed to theoretical Planck-scale dimensions. If the argument that mathematically follows from the classical GR equation were valid, then the escape velocity would never reach light speed and there would be no "imaginary" limit to distortion. The distortion would increase following a square-root curve, never reaching infinity. Bosons would decrease in both speed and energy and would increase in number because of matter/energy conservation. The density would be limited only by the Planck dimensions. So arises the first purely theoretical postulate (unlike the others, which were directly reasoned from current theory/equations) presented in this paper. If the particles formed through compression and relativistic effects via energy-to-matter/antimatter conversion were always exactly balanced, then neither flavour could achieve the matter dominance that we observe in our local universe. Particles created from compressed/slowed boson substances would continually annihilate each other in exactly balanced numbers.

So hypothesize that either flavour can act as a nuclear catalyst for the creation of more particles of its own type. Considerably more analysis and research are required to confirm the details of this postulate. However, it is reasonable to theorize that either matter or antimatter promotes the conversion from energy into more of the same particles. Under the assumption of an infinite reality, it is not even necessary to establish a density, number, or mass of particles required for the state of nuclear catalysis to consistently ensure the production of more particles of the same matter flavour. The infinity proposition does imply the process must eventually occur. It also implies there could be other antimatter realities that we have not currently detected. It is even consistent with current suggestions that other observable galaxies may consist of antimatter [15].

This researcher refuses to make any declarations on the issue, though admitting his personal leanings are towards matter/antimatter stellar organization on our observable local universe scale. All currently observed bodies define the absolute minimum size of our local matter universe, though not necessarily its full extent. In an Infinite Cosmos, the proposal put forward in this paper does not preclude the possible existence of local antimatter universes. A resolution to the question of whether the matter vs. antimatter conflict is settled at the galactic or local-universe scale may take decades, centuries or millennia of research and theorizing. Under the infinity 
proposition, it is uncertain whether this question can even be answered with absolute scientific, mathematical worth, though this writer admits absolute ignorance on the issue.

\section{Inconsistent Distance and Approach of the M31 Andromeda Galaxy}

The only data available that reflect the expansion of our local universe are the observed red shifts of objects at intergalactic distances. However, an inconsistency in these data is the approaching velocity of the Andromeda Galaxy [M31]. Its distance from the Milky Way (MW) is $7.8500 \sim 00 \mathrm{E} 2$ kiloparsecs [16]. So its distance in Megaparsecs [M31_Dist ${ }_{\mathrm{MPc}}$ ] is $7.8500 \sim 00 \mathrm{E}-1 \mathrm{Mpc}$.

The Hubble constant $\left[\mathrm{H}_{0}\right][17]$ is currently believed to be

$$
\mathrm{H}_{0}=69.3200 \sim 00(\mathrm{~km} / \mathrm{s}) / \mathrm{Mpc}
$$

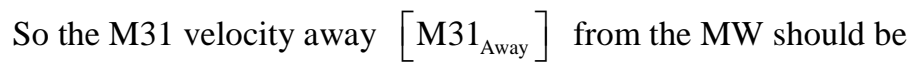

$$
\begin{gathered}
\text { M31 } 1_{\text {Away }}=\text { M31 } 1_{\text {Distance_Mpc }} * \mathrm{H}_{0} \\
\text { M31 } 1_{\text {Away }}=6.93200 \sim 00 \mathrm{E} 1((\mathrm{~km} / \mathrm{s}) / \mathrm{Mpc}) * 7.8500 \sim 00 \mathrm{E}-1 \mathrm{Mpc} \\
\mathrm{M} 31_{\text {Away }}=5.4416200 \sim 00 \mathrm{E} 1(\mathrm{~km} / \mathrm{s})
\end{gathered}
$$

The real velocity of M31 is the opposite: an approach MW velocity [18] of $301 \pm 1 \mathrm{~km} / \mathrm{s}\left[\mathrm{M} 31_{\text {Approach }}\right]$. The argument could be made that some separating velocity between the two bodies was introduced in the early moments of the Universe, but for the simple fact that the mass of M31 [18] is thought to be approximately equivalent to that of the MW, which is 1.0E12 that of Solar $_{\text {Mass }}$.

As the estimated [ Solar $_{\text {Mass }}$ ] is $1.9885 \mathrm{E} 30 \mathrm{~kg}$ [9], both Galaxies have the following mass:

$$
\begin{gathered}
\mathrm{MW}_{\text {Mass }}=\text { Solar }_{\text {Mass }} * \mathrm{MW}_{\text {Solar_Masses }_{\text {_ }}} \\
\mathrm{MW}_{\text {Mass }}=1.988500 \sim 00 \mathrm{E} 30 * 1.0 \mathrm{E} 1200 \sim 00 \\
\mathrm{MW}_{\text {Mass }}=1.9885 \mathrm{E} 42 \mathrm{~kg}
\end{gathered}
$$

Because a parsec (pc) is equal to 3.08568E16 m [19], a Megaparsec [Mpc] is 3.08568E22 m. Thus, the distance between M31 and the MW [M31_Dist] is

$$
\begin{gathered}
\text { M31_Dist }=\text { M31_ } \text { Dist }_{\text {Mpc }} * \text { Mpc } \\
\text { M31_Dist }=7.8500 \sim 00 \mathrm{E}-1 \mathrm{Mpc} * 3.0856800 \sim 00 \mathrm{E} 16 \mathrm{~m} / \mathrm{Mpc} \\
\text { M31_Dist }=2.422258800 \sim 00 \mathrm{E} 22 \mathrm{~m}
\end{gathered}
$$

Hence, the escape velocity $\left[\mathrm{MW}_{\mathrm{Esc}}\right]$ between the two bodies is

$$
\begin{gathered}
\mathrm{MW}_{\mathrm{Esc}}=\left(2 * G * \mathrm{MW}_{\text {Mass }} / \mathrm{M} 31 \_ \text {Dist }\right)^{0.5} \\
\mathrm{MW}_{\mathrm{Esc}}=(2 * 6.67384 \mathrm{E}-11 * 1.98850 \mathrm{E} 42 \mathrm{~kg} / 2.422258800 \sim 00 \mathrm{E} 22 \mathrm{~m})^{0.5 ?} \\
\mathrm{MW}_{\mathrm{Esc}}=1.04691160385258652287668852191755574063384243119569 \mathrm{E} 5 \mathrm{~m} / \mathrm{s} .
\end{gathered}
$$

The ratio of the approach velocity to the escape velocity [ Ratio $\left._{\text {Esc_to_Approach }}\right]$ is

$$
\begin{gathered}
\text { Ratio }_{\text {Esc_to_Approach }}=\mathrm{MW}_{\text {Esc }} / \mathrm{M}_{\text {Approach }} \\
\text { Ratio }_{\text {Esc_to_Approah }}=3.01000 \sim 00 \mathrm{E} 5 \mathrm{~m} / \mathrm{s} / 1.0469 \sim 569 \mathrm{E} 5 \mathrm{~m} / \mathrm{s} \\
\text { Ratio }_{\text {Esc_to_Approach }}=2.87512335227094486433516648533090714315790464528012 \mathrm{E} 0
\end{gathered}
$$

Thus, M31 is approaching the MW at more than 5/2 times their mutual escape velocity, in an "expanding" universe. In fact, the escape velocity is likely even less than the above value. The distance that should be used in the escape equation may be greater; because the relevant distance is the distance between the centres of gravity of the two objects. However, both objects are so disperse that the true location of the centre of gravity is a debatable issue. The above velocity is the absolute maximum escape velocity; the objects may be mutually ap- 
proaching at a much greater proportion of the Real value that would be generated by the interaction of two such disperse objects. Is this not another argument for frequency decay due to the local universe Cosmosphere explanation, as opposed to a Hubble reality? M31 is simply moving towards us at a velocity too high to be overcome by a Cosmospheric frequency shift. The complete argument is made in greater detail in the following paper: Frequency Decay through Electromagnetic Radiation Absorption and Re-Emission by Inter-Galactic Dark Matter as an Alternate Explanation For the Hubble Constant. The final JHEPGC version is available on http://www.scirp.org/journal/PaperInformation.aspx?PaperID=67680. A preliminary version of that paper is available in the Alternative Archive of E-prints in Science and Mathematics at http://vixra.org/abs/1502.0107.

\section{Summary}

Similar to the Real velocity in Special Relativity, the escape velocity is limited to a maximum of the speed of light under General Relativity restrictions. This limit implies that the General Relativistic distortion could approach infinity but never reach it. The effects derived from current relativistic theory would be unable to bring about an "imaginary” state of reality. The Real gravity of any body could never do more than approach infinity. Relativistic effects would reduce the gravity, though not to any definable value. The purpose of this paper was not to dismiss the value of relativistic theory but rather to identify some of the incompletion in its mathematical reasoning. This argument is reinforced by observation of the Sagittarius A phenomenon at the centre of the Milky Way. The points presented in this paper will be argued much more thoroughly and mathematically in subsequent papers, which will discuss how relativistic effects would be experienced from the perspective of the moving/distorted object.

\section{References}

[1] Bailey, J. (1977) Measurements of Relativistic Time Dilatation for Positive and Negative Muons in a Circular Orbit. Nature, 268, 301-305. http://www.nature.com/nature/journal/v268/n5618/abs/268301a0.html

[2] Penzias, A.A. and Wilson, R.W. (1965) A Measurement of Excess Antenna Temperature at 4080 Mc/s. Astrophysical Journal, 142, 419-421

[3] UNIVERSEFORUM-Produced for NASA for the Harvard Smithsonian Center for Astrophysics. Where Did the Universe Come from? https://www.cfa.harvard.edu/seuforum/bb_whycare.htm

[4] Antimatter at CERN. http://home.cern/topics/antimatter/antimatter-cern

[5] Hubble Space Telescope. Galaxy Cluster Abell 2261. http://www.nasa.gov/mission pages/hubble/science/a2261-bcg.html

[6] Special Relativity Aces Time Trial. Nature. http://www.nature.com/news/special-relativity-aces-time-trial-1.15970

[7] National Institute of Standards and Technology. Hydrogen (H). http://physics.nist.gov/PhysRefData/Handbook/Tables/hydrogentable1.htm

[8] National Institute of Standards and Technology, Physical Meas. Laboratory. Iron (Fe). http://physics.nist.gov/PhysRefData/Handbook/Tables/irontable1.htm

[9] Williams, D.R. Sun Fact Sheet. NASA. http://nssdc.gsfc.nasa.gov/planetary/factsheet/sunfact.html

[10] Gillies, G.T. (1997) The Newtonian Gravitational Constant: Recent Measurements and Related Studies. Reports on Progress in Physics, 60, 151-225. http://dx.doi.org/10.1088/0034-4885/60/2/001

[11] Eisenhauer, F., et al. (2003) A Geometric Determination of the Distance to the Galactic Center. The Astrophysical Journal, 597, . http://dx.doi.org/10.1086/380188

[12] Gillessen, S., et al. (2009) Monitoring Stellar Orbits around the Massive Black Hole in the Galactic Center. The Astrophysical Journal, 692, 1075-1109. http://dx.doi.org/10.1088/0004-637X/692/2/1075

[13] The NIST Reference on Constants, Units and Uncertainty; Planck Length. http://physics.nist.gov/cgi-bin/cuu/Value?plkl

[14] Close, F.E. (2009) Antimatter. Oxford University Press US, New York, p. 114.

[15] McConnachie, A.W., Irwin, M.J., Ferguson, A.M.N., Ibata, R.A., Lewis, G.F. and Tanvir, N. (2005) Distances and Metallicities for 17 Local Group Galaxies. Monthly Notices Royal Astronomic Society, 356, 979-997. http://dx.doi.org/10.1111/j.1365-2966.2004.08514.x http://mnras.oxfordjournals.org/content/356/3/979.full?sid=1a570508-7670-42a8-af78-b4879a80e7cb

[16] McConnachie, A.W., Irwin, M.J., Ferguson, A.M.N., Ibata, R.A., Lewis, G.F. and Tanvir, N. (2005) Distances and 
Metallicities for 17 Local Group Galaxies. Monthly Notices Royal Astronomic Society, 356, 979-997. http://dx.doi.org/10.1111/j.1365-2966.2004.08514.x

http://mnras.oxfordjournals.org/content/356/3/979.full?sid=1a570508-7670-42a8-af78-b4879a80e7cb

[17] Nine-Year Wilkinson Microwave Anisotropy Probe (WMAP) Observations: Final Maps and Results. http://arxiv.org/pdf/1212.5225v3.pdf

[18] Karachentsev, I.D. and Kashibadze, O.G. (2006) Masses of the Local Group and of the M81 Group Estimated from Distortions in the Local Velocity Field. Astrophysics, 49, 3-18. http://dx.doi.org/10.1007/s10511-006-0002-6

[19] Dictionary of Geophysics, Astrophysics, and Astronomy. CRC Press, Boca Raton. http://www.deu.edu.tr/userweb/emre.timur/dosyalar/Dictionary\%20of\%20Geophysics,\%20Astrophysics\%20and\%20A stronomy.pdf

\section{Submit or recommend next manuscript to SCIRP and we will provide best service for you:}

Accepting pre-submission inquiries through Email, Facebook, Linkedin, Twitter, etc A wide selection of journals (inclusive of 9 subjects, more than 200 journals)

Providing a 24-hour high-quality service

User-friendly online submission system

Fair and swift peer-review system

Efficient typesetting and proofreading procedure

Display of the result of downloads and visits, as well as the number of cited articles

Maximum dissemination of your research work

Submit your manuscript at: http://papersubmission.scirp.org/ 